\title{
Comparison of Glucose and HBA1C Values Between Fin- ger-Piercing and Continuous Glucose Monitoring Sensor Using Gh-Method: Math-Physical Medicine (no. 293)
}

\author{
Gerald C Hsu* \\ University-eclaireMD Foundation, USA
}

\begin{abstract}
This article discusses the fundamental characteristics of measured glucose levels and predicted glycated hemoglobin A1c (HbA1c) values among three sets of collected data, measured finger-piercing and continuous glucose monitoring (CGM) sensor device collected glucose levels at 15-minute (15-min) and 5-minute (5-min) intervals. The average glucose (in milligram per deciliter-mg/dL) is listed below:
\end{abstract}

Finger glucose: $109 \mathrm{mg} / \mathrm{dL}(100 \%)$

Sensor at 15-min: $120 \mathrm{mg} / \mathrm{dL}$ (109\%)

Sensor at 5-min: $117 \mathrm{mg} / \mathrm{dL}(107 \%)$

Using candlestick chart, the comparison of average glucoses during this period between two sensor glucose (mg/dL) data (15-min/5-min) are as follows:

Open glucose: 108/111

Close glucose: $115 / 115$

Maximum (max) glucose: $170 / 175$

Minimum (min) glucose: 85/83

Average glucose: $120 / 117$

Additional analysis of time above range (TAR) $\geq 140 \mathrm{mg} / \mathrm{dL}$ for hyperglycemia, time within the range (TIR) from $70-140 \mathrm{mg} / \mathrm{dL}$ for normal, time below range (TBR) $\leq 70 \mathrm{mg} / \mathrm{dL}$ for hypoglycemia based on two sensor candlesticks revealing the following information in a specific format of TAR\%/ TIR\%/TBR\%.

\section{5-min:18.3\%, 80.5\%, 1.2\%}

5-min: $17.0 \%, 81.9 \%, 1.1 \%$

By evaluating the results of the TIR analysis, the 5-min glucose levels appear to be marginally healthier (1.4\%) than the 15-min ones. During the coronavirus pandemic (COVID 19) quarantine period, the author lived a rather unique lifestyle which is extremely calm with regular routines, such as eating home-cooked meals and exercising on a regular basis. As a result, his $\mathrm{HbA1c}$ has decreased from $6.6 \%$ to $6.3 \%$ with an average A1c of $6.4 \%$ without taking any diabetes medications. However, these three different measurement methods still provide three different sets of glucoses levels which are within a $10 \%$ margin of differences, while the HbA1c values are particularly close to each other between the finger-piercing and CGM 15-min.

Keywords: Glucose, Finger-piercing, Candlestick chart, Glycated hemoglobin A1c

\begin{tabular}{|l|l|}
\hline Quick Response Code: & *Corresponding author: Gerald C Hsu, University-eclaireMD Foundation, USA \\
Received: 24 November, 2020 & Published: 01 March, 2021 \\
\hline & Citation: Gerald C Hsu. Comparison of Glucose and HBA1C Values Between Finger-Piercing \\
and Continuous Glucose Monitoring Sensor Using Gh-Method: Math-Physical Medicine (no. \\
293). Curr Inv Cln Med Res. 2021;1(1):1-6. DOI: 10.53902/CICMR.2021.01.000503
\end{tabular}




\section{Introduction}

This article discusses the fundamental characteristics of measured glucoses and predicted HbA1c values among three sets of collected data, measured finger-piercing (finger) and CGM sensor device collected glucoses at 15-minute (15-min) and 5-minute (5min) intervals.

\section{Methods}

The author has been a severe type 2 diabetes (T2D) patient for over 25 years. Starting about 8 years ago, he used the finger-piercing method to measure his glucose levels four times daily: once in the early morning and three times 2 hours after each meal. Aproximately 2 years ago, he applied a CGM device (Libre sensor-it is necessary to mention the producer and city) on his upper arm and collected his glucose levels approximately 80 times per day. Furthermore, starting on the quaratine period beginning, he attached a Bluetooth device on the CGM sensor to collect about 240 samples per day at 5-min intervals. The purpose of this device was to investigate the damages performed on his internal organs resulting from the higher frequency with lower amplitude glucose components. Therefore, in total, he has collected 12,448 finger data within 3,112 days, 63,840 15-min data within 798 days, and 34,320 5-min data within 143 days. In summary, he has a total of 110,608 glucose data from the three measurement groups: finger, 15-min, and 5-min.

He developed a mathematical model of the predicted HbA1c value known as "Daily A1c N-2" which uses his collected glucose data as input and the lab-tested HbA1c as its A1c data calibration. His predicted $\mathrm{HbA1c}$ formula is defined by using glucose from the previous four months.
The previous fourth month counts for $10 \%$, the third previous month counts for $15 \%$, the second previous month counts for $25 \%$, and first month counts for $50 \%$. The author has tried many different arithmetic models and found that this Daily A1c N-2 model's results would fit the best with the lab-tested A1c values.

A Japanese merchant, who traded in the rice market in Osaka, Japan, started the candlestick charting around 1850. An American, Steve Nison brought the "candlestick model" to the Western world in 1991. These techniques are largely used in today's stock market to predict the price direction or action. The author had the idea to study glucose behavior by using the candlestick chart (aka "K-Line") and subsequently developed a customized software to analyze his big glucose data ${ }^{1,2}$ In his K-Line model, he used the following five key values, i.e. "open" (starting), "close" (ending), "maximum", "minimum", and "average" to describe the glucose fluctuations over the course of a day. Obviously, this candlestick model cannot be applied on finger glucoses data due to its small data set of having only 4 data per day.

\section{Results}

Initially, the author selects the period of 142 days, as his investigation period. This particular period contains all of his three glucose groups, finger, 15-min, and 5-min. This time period is sufficient for displaying and analyzing glucose behaviors. However, for analyzing his predicted $\mathrm{HbA1c}$, in order to obtain a complete and reliable calculated results, he must ignore the first four months of the $\mathrm{HbA1C}$ due to his original design of the predicted $\mathrm{HbA1C}$ equation based on the four previous months of glucose data.

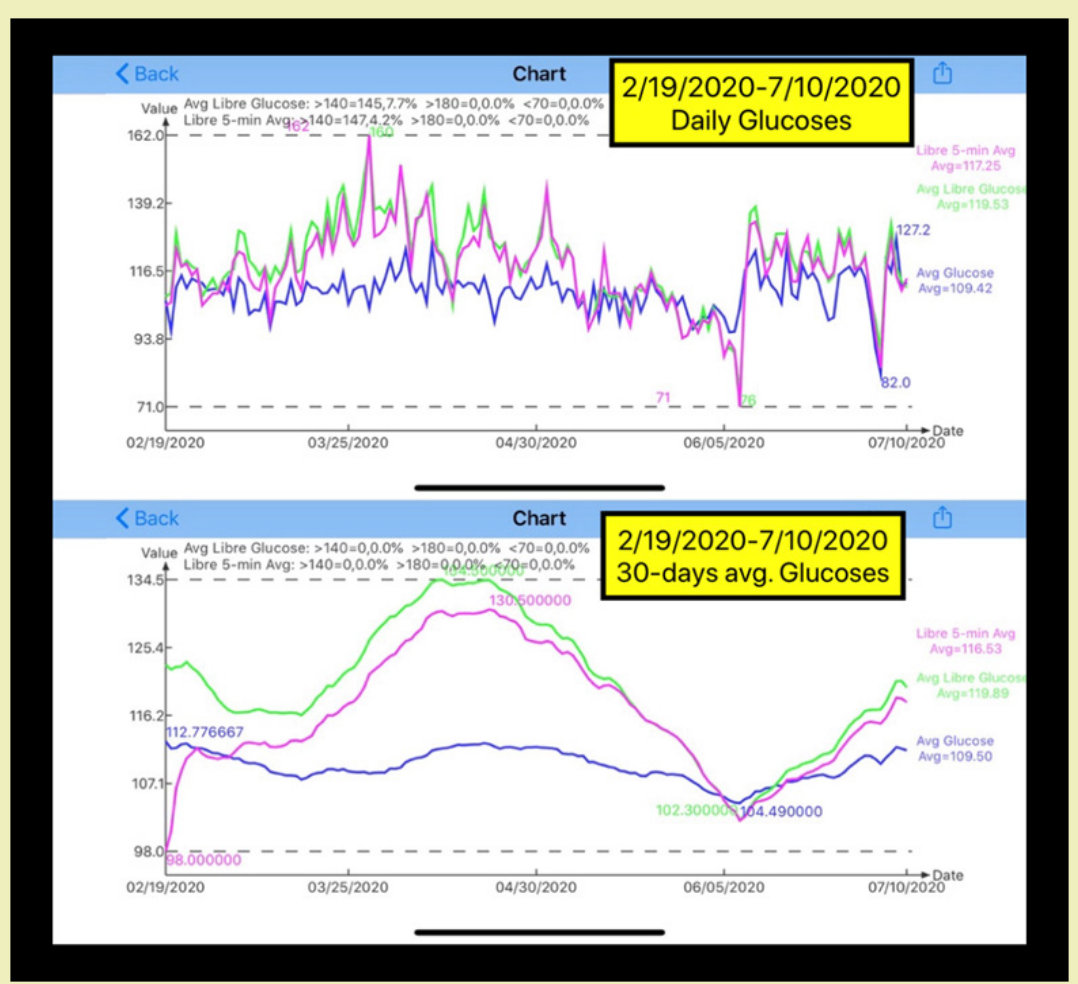

Figure 1: Daily glucoses of three measurement methods. 
Figure 1 shows both daily glucose data curve and 30-days moving average glucose data curve for all three groups. The average glucose are listed:

Finger: 109 mg/dL (100\%)

\section{5-min: $120 \mathrm{mg} / \mathrm{dL}$ (109\%)}

\section{5-min: $117 \mathrm{mg} / \mathrm{dL}(107 \%)$}

Further detailed examination of these three waveforms (i.e. curves) reveals two additional facts. First, the curve shapes or waveforms of the 5-min and 15-min are remarkably similar except that the 5-min wave contains more higher-frequency glucose components which would be useful for the author's further research work. Second, during the segment from March to May, about $60 \%$ of the total timespan of this investigation period, the sensor glucoses values (both 5-min and 15-min) are higher than finger ones. This observed phenomenon is clearly illustrated in the bottom figure of the 30-days moving average. The reasons for this could be caused by different time instants of measuring finger glucose or the sensor device's reliability issues3. It should be noted that the first 30-days segment of the moving average curve should be disregarded due to the 5-min data collection starting on 2/19/2020.

Figure 2 depicts the K-Line diagram of both 15-min and 5-min. The finger glucoses values have only 4 data per day; therefore, it has no relevance to the $\mathrm{K}$-Line on finger data.

Here is the definition of the five characteristic values for a candlestick:

Open glucose: starting at 7:00

Close glucose: ending at 23:30

Max glucose: highest value

Min glucose: lowest value

Average glucose: daily average

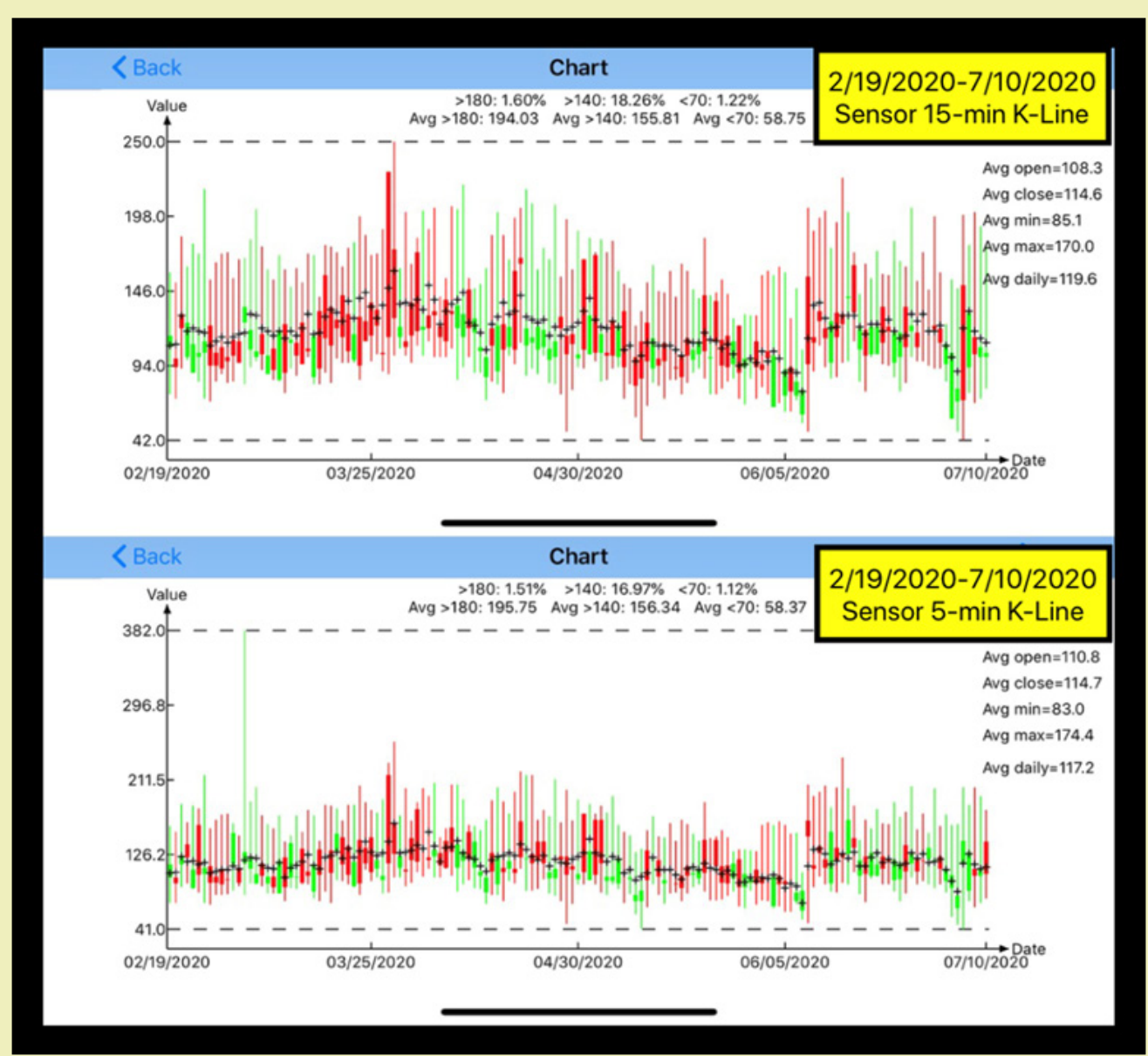

Figure 2: Candlestick glucoses of three measurement methods.

The comparison of average glucoses during this period between two sensor glucose (in mg/dL) data (15-min/5-min) are as follows:

Open glucose: $108 / 111$

Close glucose: $115 / 115$

Maximum glucose: $170 / 175$
Minimum glucose: 85/83

Average glucose: 120/117

The max glucose of the 5-min is higher than the 15-min, while the min glucose of the 5-min is lower than the 15-min due to the 5-min measurement capturing more data, including extremities. 
The average of the 15-min is higher than 5-min due to the 5-min measurement catching more lower glucoses levels than the 15-min (see TIR analysis below).

A further deeper analysis of $\mathrm{TAR} \geq 140 \mathrm{mg} / \mathrm{dL}$ for hyperglycemia, TIR from 70-140 mg/dL for normal, and TBR $\leq 70 \mathrm{mg} / \mathrm{dL}$ for hypoglycemia based on the two sensor candlesticks revealed the following information in a specific format of TAR\%/TIR\%/TBR\%.

15-min: $18.3 \%, 80.5 \%, 1.2 \%$

\section{5-min: $17.0 \%, 81.9 \%, 1.1 \%$}

By evaluating the results of the TIR analysis, the 5-min glucoses data appear to be marginally healthier (1.4\%) than the 15 -min glucoses values.

Figure 3 reflects the author's mathematically predicted HbA1c results for these three glucoses. Due to his predicted $\mathrm{HbA1}$ c requirement of the four previous months glucose data for his calculation and this entire investigation period is less than 5 months (only 142 days), he will not discuss the HbA1C of the 5-min even though its curve is shown in this figure. His finger $\mathrm{A} 1 \mathrm{c}$ is $6.43 \%$ and his $15-\mathrm{min}$ $\mathrm{A} 1 \mathrm{C}$ is $6.39 \%$. If he adopted the lab-tested first digit display format, then both of his finger and 15-min HbA1C are 6.4\%. The author has conducted his research on accuracy of his predicted HbA1c4 over 8 sub-periods with 5-months each using his weight, diet, and exercise data to achieve the $100 \%$ prediction accuracy. Therefore, during this investigation period along with the COVID-19 quarantine timeframe, he was unable to go to a hospital or a laboratory to conduct the HbA1c test. He decided to use this mathematically predicted $\mathrm{HbA} 1 \mathrm{c}$ of $6.4 \%$ as his base for the research work.

In Figure 3, Finger A1c decreased from 6.6\% in mid-February to $6.5 \%$ by the end of April and was $6.3 \%$ in early June. The Sensor 15-min A1c reached $6.7 \%$ by the end of April and then dropped to $5.9 \%$ in early June; however, the average HbA1c for both finger and sensor 15 -min are $6.4 \%$.

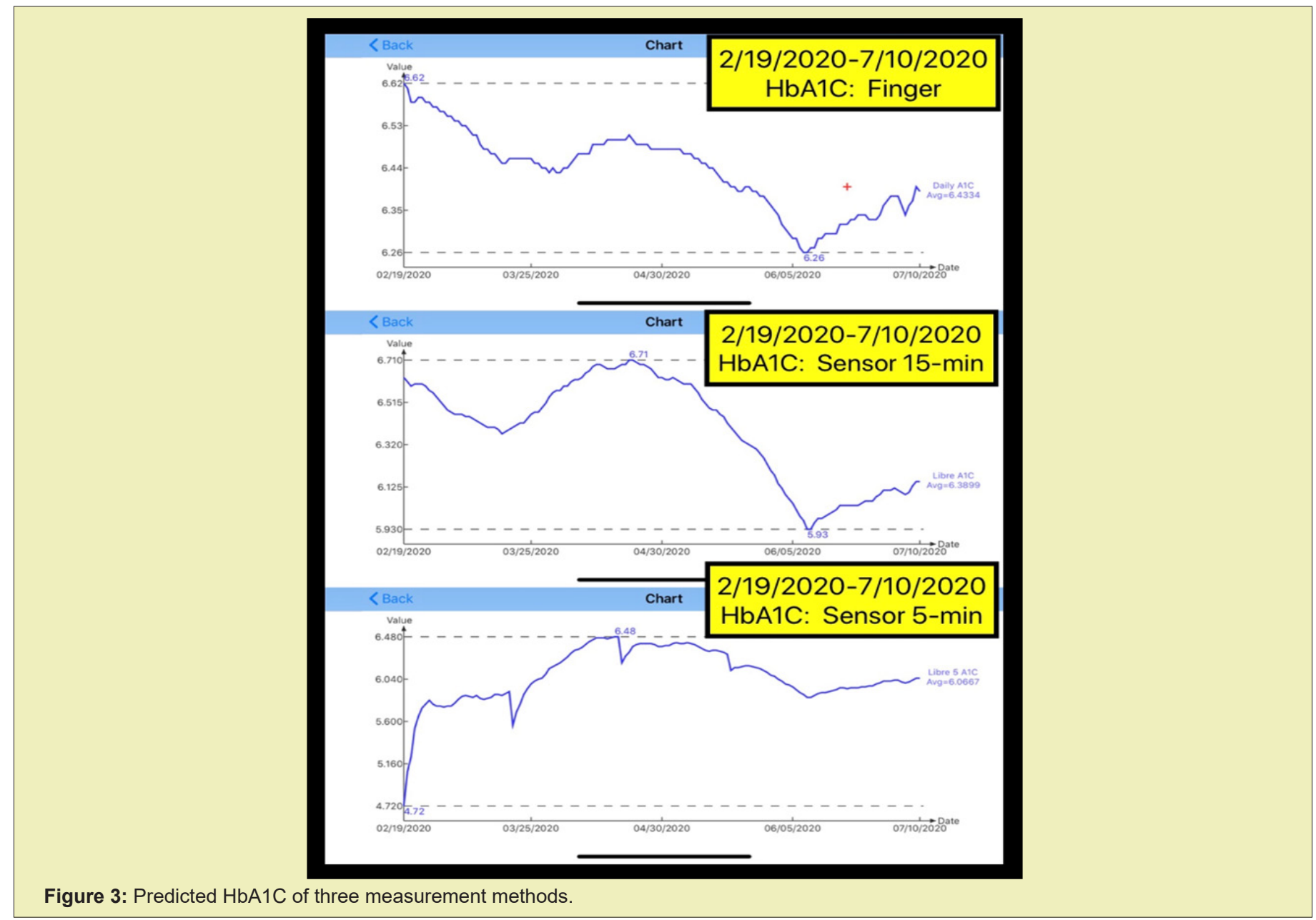

Figure 4 displays the relationship between the 30-days moving average glucose and predicted HbA1c. The top diagram is the $30-$ days moving average glucose of finger versus 15-min which has a correlation coefficient of $82 \%$. The second diagram is the predicted $\mathrm{HbA} 1 \mathrm{c}$ of finger versus 15-min which has a correlation coefficient of $86 \%$. Both correlation coefficients are above $80 \%$ which mean these two sets of data (finger versus sensor 15-min) have extremely similar wave patterns. The third and bottom diagrams show specific views of combining the 30-days moving average glucose and A1c curves for both finger and sensor 15-min together (i.e.,disregard the scale of y-axis). In this way, it is easier to see the relative shapes of the two curves. The data amount collected daily are dif- 
ferent with 4 data per day for finger and 80 data per day for Sensor 15-min. Fundamentally, both glucose waveforms are remarkably comparable, except for each measurement method would yield a different "absolute" value, specifically at the peak glucose value and the lowest glucose value. ${ }^{1-4}$

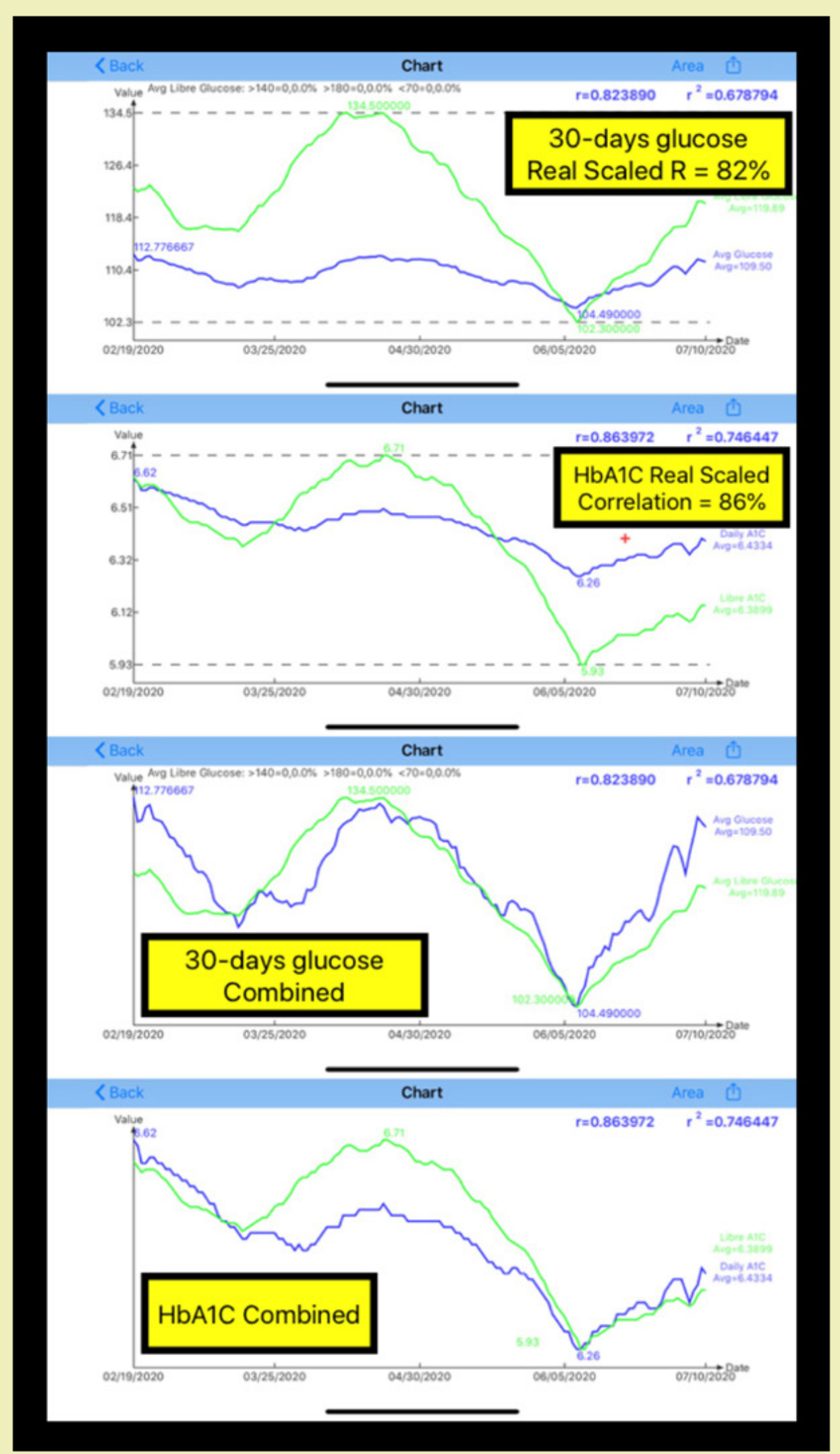

Figure 4: Glucose \& HbA1C for real-scaled and combined. 


\section{Conclusion}

During this COVID-19 quarantine period, the author lived a rather unique lifestyle which is extremely calm with regular routines, such as eating home-cooked meals and exercising on a regular basis. As a result, his $\mathrm{HbA1c}$ has decreased from $6.6 \%$ to $6.3 \%$ with an average A1c of $6.4 \%$ without taking any diabetes medications. However, these three different measurement methods still provide three different sets of glucoses levels which are within a 10\% margin of differences, while HbA1c values are particularly close to each other between finger and 15-min.

\section{Acknowledgments}

None.

\section{Funding}

None.

\section{Conflicts of Interest}

Author declares that there is no conflict of interest.

\section{References}

1. Hsu Gerald C. Using Candlestick Charting Techniques to Investigate Glucose Behaviors via GH-Method: Math-Physical Medicine (No. 76). The New American Journal of Medicine. 2020;V1(2):1-4.

2. Hsu Gerald C. Comparison study of PPG characteristics from candlestick model using GH-Method: Math-Physical Medicine (No. 261). J Nutr Diet Pract. 2020;4(3).

3. Hsu Gerald C. A case study of the impact on glucose, particularly postprandial plasma glucose based on the 14-day sensor device reliability using GH-Method: math-physical medicine (No. 124). J B Bio Engine. 2020;1(1).

4. Hsu Gerald C. Investigation of HbA1C variances and predictions over eight sub-periods using GH-Method: math-physical medicine (No. 279). 